\title{
MICROALBUMINURIA AS A PROGNOSTIC VALUE FOR POST-OPERATIVE PATIENTS WITH RISK OF SEPSIS AFTER MAJOR ABDOMINAL SURGERY
}

\author{
Sameh Hamdy Seyam, MD., Mostafa Mohammed Sabra, MD., Mohsen Mohsen Eissa, MD., \\ Mofeed Abdalla Abdelmaboud, MD., Tawfik Mohamed Nooreldin, MD. \\ Anesthesia and ICU Department; Faculty of Medicine; Al-Azhar University; Egypt.
}

\begin{abstract}
Background:- Microalbuminuria is one of the early indicators of sepsis. It is caused by diffused endothelial dysfunction in critically ill septic patients. The degree of microalbuminuria corresponds with the intensity of the inflammatory contempt.

Purpose:- To investigate whether microalbuminuria has a prognostic value in surgical sepsis or not, with particular regard to its role in anticipating illness intensity, clinical course and mortality.

Methods:- The study was applied in the critical care unit, Sayed Galal hospital, Faculty of medicine, Al-Azhar university. A sum of 80 patients were included in a prospective, non-interventional study following major abdominal surgery, and divided into two groups each group containing 40 patients. Albumin/Creatinine ratio (ACR, mg/g) was measured in urinary samples collected preoperatively (tp), upon ICU admission (t0), after 6 hours (t6) and at 24 hours (t24). Acute physiology and chronic health evaluation II (APACHE II) score was calculated 24, 48 and 72 hours, and Sequential Organ Failure assessment (SOFA) score was calculated daily for 3 days. Clinical outcome (length of ICU stay), and final outcome (survival or mortality rates) were recorded and documented for all patients.

Results:- There was statistically significant difference between groups according to ACR t0 to t24 specially at t6 which was highly significant in group II in relation to group I with p-value $<0.001$. There was highly statistically significant difference between groups according to APACHE score in group II in relation to group I for APACHE 24, 48, and 72, p-value $<0.001$. There was highly statistically significant difference between groups according to SOFA score in group II in relation to group I for SOFA1,2 and 3, p-value $<0.001$. There was statistically significant difference between groups according to outcome (death was $12.5 \%$ in group I in relation to $37.5 \%$ in group II) p-value 0.020 .
\end{abstract}

Conclusion:- Microalbuminuria an easy, rapid and simple tool has a prognostic value in surgical sepsis, with precise regard to its role in anticipating illness severity, clinical course and mortality.

Key words:- Microalbuminuria; APACHE score; SOFA score; mortality; major abdominal surgery.

Corresponding Author

Name: Sameh Hamdy Seyam

Tel.: 01202942446

\section{INTRODUCTION}

$\mathrm{n}$ intensive care environments, anticipating of outcome of patients is of crucial importance to the intensivist. It allows deciding of early therapeutic maneuvers, optimum resource allotment and appropriate advising of the family or patient ${ }^{[1]}$.

A number of prognostic tools have been created for the above target. The two main widely accepted systems to predict mortality are the Simplified Acute Physiology Score (SAP) II scores and the Acute Physiology and Chronic Health Evaluation (APACHE) ${ }^{[1]}$.

Previously, microalbuminuria was used to anticipate the assault of renal impairment in

\section{Received: February 2016}

Accepted: April 2016

diabetic patients. Recently, it has shown as an early predictor of disease intensity in many acute inflammatory conditions ${ }^{[2]}$.

Another important concern, it has been found to be an early predictor of mortality in different groups of critically ill patients ${ }^{[3]}$. Additionally, it has been known as a useful predictor of cardiovascular events in adults ${ }^{[4]}$.

By definition, Microalbuminuria is a urinary albumin concentration of $30-200 \mathrm{mg}$ litre- ${ }^{1}$.It is exhibited as the urinary albumin to creatinine ratio (ACR) to correct for alterations in urine output ${ }^{[3]}$.

The cause of microalbuminuria in postoperative surgical patients most probably 
related to global profound endothelial dysfunction arising from the effects of inflammatory mediators, released during the severe inflammatory assaults that are conjoined with critical illnesses leading to systemic increase in capillary permeability ${ }^{[15]}$.

It is one of the common readings in critically ill patients, where it has demonstrated not only as a predictor of organ impairment and vasopressor/inotropic requirements but also of mortality, being superior to the Sequential Organ Function Assessment (SOFA) score and Acute Physiological and Chronic Health Evaluation (APACHE)II score ${ }^{[5]}$.

\section{AIM OF THE WORK}

To investigate whether microalbuminuria has a prognostic value in surgical sepsis or not, with special regard to its aspect in anticipating illness severity, clinical course and primary outcome (sepsis) or secondary outcome (mortality).

\section{PATIENTS AND METHODS}

Patients; this study was approved by the medical Ethics committee at Faculty of Medicine, Al-Azhar University. We prospectively enrolled 80 patients. We divided the patients into two subgroups according to significant rise of microalbumin at 6 hours post-operatively ( group I with no significant microalbuminuria and group II with significant microalbuminuria at t6), each group of 40 patients. Patients were sequentially selected following total gastrectomy, Whipple-resection of the pancreas, elective oesophagectomy and liver resection due to tumour removal, admitted to Critical care unit, Sayed Galal hospital, Faculty of medicine, Al-Azhar university over ten months period from March 2015 to January 2016. Informed consent given by the patient or immediate relative (first degree). This study did not interfere with the current medical practice of the investigator. No invasive medical procedure is required by the study.

Inclusion criteria: 1) Age above 18 years 2) Sepsis (Society of Critical Care Medicine "SCCM" criteria) of postoperative onset suspected by the treating physician or confirmed infection + criteria of SIRS.

3) SIRS (SCCM criteria) ${ }^{[6]}$ was diagnosed when there were two or more of the following: Heart rate $(\mathrm{HR})>90 / \mathrm{min}$, Respiratory rate (RR) > 20/min or $\mathrm{PaCO} 2<32 \mathrm{mmHg}$, Temperature $>38^{\circ} \mathrm{C}$ or $<36^{\circ} \mathrm{C}$, White blood cell count $>12,000 / \mathrm{mm}^{3}$ or $<4000 / \mathrm{mm}^{3}$ or $>10 \%$ immature neutrophils.

Exclusion criteria included patients with ischemic heart disease, hypertension, diabetes or with concurrent treatment with any of the antiproteinuric drugs, (Angiotensin-converting enzyme inhibitors (ACE-I), Angiotensin II, angiotensin I receptor blockers (ARBs), lipid lowering drugs such as statins or fibrates, and recently also oral glycosaminoglycane sulodexide), patients with chronic renal disease (serum creatinine level $\geq 2.0 \mathrm{mg} / \mathrm{dL}$ ) or on replacement therapy (dialysis), no urine output on the first day of admission, hospital stay less than 48 hours,

Patients who did not met any of the exclusion criteria were selected randomly and prospectively included into the study on the day of ICU admission and they were followed up for an ultimate period of 25 days or till the day of discharge or demise.

\section{Evaluation of Patients:-}

\section{Physical and laboratory examination:}

1) Full Clinical Evaluation: Including history and physical examination with special emphasis on vital signs (Heart Rate "HR", Blood Pressure "BP", Respiratory Rate "RR" and Temperature "Temp.") and Glasgow Coma Scale (GCS); which were evaluated at the preoperative time (tp), postoperative (t0), 6 hours postoperative (t6), 24 hours postoperative (t24), 48 hours postoperative (t48), and 72 hours postoperative (t72).

\section{2) Laboratory investigations:}

- Routine Labs: complete blood count (CBC): Hemoglobin, Hematocrit, White blood cells and platelet count, arterial blood gases (ABGs), Liver function tests: Alanine aminotransferase (ALT), Aspartate aminotransferase (AST), bilirubin (BIL) and albumin, Kidney Function 
Tests: $\mathrm{Na}, \mathrm{K}$, Creatinine and Urea, and random blood sugar, Coagulation profile: prothrombin time (PT), prothrombin concentration (PC), International Normalization Ratio (INR) and partial thromboplastin time (PTT).

These routine Labs were withdrawn on study day 1 and then daily for 3 days.

- Labs specific for our study: The reading will be exhibited as the urinary albumin / creatinine ratio (ACR) to correct the alterations in urine output and were referred to as $\mathrm{ACR}^{[7]}$.

Spot urine samples taken at the preoperative time (tp), postoperative (t0), 6 hours postoperative (t6), 24 hours postoperative (t24), 48 hours postoperative (t48), and 72 hours postoperative ( $\mathrm{t} 72)$.

Urine samples were sent to the biochemistry lab and stored at $-20^{\circ} \mathrm{C}$ till the time of analysis. Urinary creatinine concentration was checked by modified kinetic Jaffe reaction (Dimension RxL Max, Dade Behring Inc., U.S.A) while Urinary microalbumin concentration was checked by the immunoturbidimetric method [8].

The methods covered an analytical spectrum of $0-20 \mathrm{mg} / \mathrm{dl}$ for creatinine and 1.3$100 \mathrm{mg} / \mathrm{L}$ for microalbumin. Microalbuminuria was expressed by ACR values between 30 and $299 \mathrm{mg} / \mathrm{g}$ creatinine. Clinical proteinuria is considered if ACR of $>300 \mathrm{mg} / \mathrm{g}$ creatinine. Healthy human has ACR $<30 \mathrm{mg} / \mathrm{g}$ creatinine $^{[7]}$. A value of 3.4 to $34 \mathrm{mg} / \mathrm{mmol}$ of creatinine i,e, 30 to $300 \mathrm{mg} / \mathrm{g}$ creatinine recommends that albumin excretion is between 30 and $300 \mathrm{mg} /$ day and accordingly microalbuminuria is most possibly present . Values above $300 \mathrm{mg} / \mathrm{g}$ creatinine (or 34 $\mathrm{mg} / \mathrm{mmol}$ ) are suggestive of macroalbuminuria. (to convert $\mathrm{mg} / \mathrm{mol}$ to $\mathrm{mg} / \mathrm{g}$, multiply by $8.84)^{[9]}$.

3) Microbiological studies: Including full septic workup (sputum, blood, urine, wound or biological fluid culture according to clinical suspicion) prior to antibiotic administration.

4) Clinical data: Length of ICU stay, primary outcome (sepsis) or secondary outcome (mortality) reported for all patients.
5) imaging studies: required to identify the source of sepsis e.g. (chest X-ray and ultrasound).

\section{6) Application scoring Systems:}

APACHE II score evaluated at 24, 48, and 72 hours later ${ }^{[10]}$.

SOFA score was evaluated on study day 1 and then daily until a total of 3 days ${ }^{[11]}$.

\section{Statistics}

Sample size justification:

MedCalc $^{\circledR}$ version 12.3.0.0 program was used for calculations of sample size, statistical calculator based on $95 \%$ confidence interval and power of the study $80 \%$ with $\alpha$ error $5 \%$, According to a previous study ${ }^{[12]}$, showed that the Microalbuminuria, explained as the urinary albumin/creatinine ratio (ACR), is a reflection of increased capillary permeability related to the systemic inflammatory response syndrome (SIRS), and has been found to be prognostic of outcome in several trials. Therefore, we explored the importance of ACR as a prognostic factor of mortality. Based on this, sample size was calculated according to these values produced a minimum of 76 cases were enough to find such a difference. Assuming a drop-out ratio of $5 \%$, the sample size will be 80 cases in the study group.

\section{Statistical analysis:}

Data were interpreted using Statistical Program for Social Science (SPSS) version 20.0. Qualitative data were explained as percent and frequency. Quantitative data were explained as mean \pm standard deviation (SD).

The following tests were done:

- Independent-samples t-test of significance was used when comparing between two means.

- Probability (P-value)

- P-value $\leq 0.05$ was considered significant.

- P-value $\leq 0.001$ was considered as highly significant.

- Chi-square $\left(\mathrm{X}^{2}\right)$ test of significance was used in order to compare proportions between two qualitative parameters. $\mathrm{P}$-value $>0.05$ was considered insignificant. 


\section{RESULTS}

I) Demographic and clinical information at ICU admission:

There was no statistically significant difference between groups according to demographic data ( $p$-value 0.309 for age, $p$-value 0.821 for sex), pathology (p-value 0.807 for gastrectomy group, p-value 0.629 for oesophagectomy group, p-value 0.848 for liver resection group, p-value 0.474 for whipple-resection group) and ASA (p-value 0.498 for ASA II and ASA III). II) Microalbuminuria in relation to $\mathrm{BMI}$ and comorbidities:-
There was no statistically significant difference between groups according to BMI ( $\mathrm{p}$-value 0.086 ) and comorbidities (p-value 0.811 for patients with no comorbidities, 0.738 for patients with DM, 0.779 for patients with HTN, 0.752 for patients with DM+HTN)

III) Microalbuminuria and length of ICU stay:-

There was statistically significant difference between groups according to ICU length of stay (LOS) (11.35 \pm 3.17 for group II in relation to $8.52 \pm 2.27$ for group I), (p-value 0.001).

Table(1): Comparison between groups according to demographic data, pathology and ASA.

\begin{tabular}{lllll}
\hline & Group I & Group II & t/x2* & p-value \\
\hline Age (years) & $52.75 \pm 7.75$ & $54.50 \pm 7.54$ & 1.024 & 0.309 \\
\hline Sex [No. (\%)] & & & \\
\hline Male & $22(55 \%)$ & $24(60 \%)$ & \multirow{2}{*}{$0.051^{*}$} & 0.821 \\
\hline Female & $18(45 \%)$ & $16(40 \%)$ & & \\
\hline Pathology[No.\% ] & & & $0.060^{*}$ & 0.807 \\
\hline$\quad$ Gastrectomy & $11(27.5 \%)$ & $13(32.5 \%)$ & $0.233^{*}$ & 0.629 \\
\hline Oesophagectomy & $11(27.5 \%)$ & $14(35 \%)$ & $0.037 *$ & 0.848 \\
\hline Liver Resection & $3(7.5 \%)$ & $2(5 \%)$ & $0.513 *$ & 0.474 \\
\hline Whipple- Resection & $15(37.5 \%)$ & $11(27.5 \%)$ & & \\
\hline II $[$ No. (\%)] & & & \\
\hline III & $19(47.5 \%)$ & $15(37.5 \%)$ & $0.460 *$ & 0.498 \\
\hline
\end{tabular}

* Chi-square test, t: Independent Sample t-test

Table (2): Comparison between groups according BMI, co-morbidities and ICU LOS.

\begin{tabular}{lllll}
\hline & Group I & Group II & t/x2* & p-value \\
\hline BMI & $26 \pm 4.6$ & $27.45 \pm 2.57$ & 1.740 & 0.086 \\
\hline Co-Morbidities & & & & \\
\hline No & $12(30 \%)$ & $14(35 \%)$ & $0.057 *$ & 0.811 \\
\hline Asthma & $14(35 \%)$ & $12(30 \%)$ & $0.113^{*}$ & 0.738 \\
\hline A OPD & $7(17.5 \%)$ & $9(22.5 \%)$ & $0.078^{*}$ & 0.779 \\
\hline ICU LOS & $7(17.5 \%)$ & $5(12.5 \%)$ & $0.098^{*}$ & 0.752 \\
\hline
\end{tabular}

* Chi-square test, t: Independent Sample t-test

IV) Microalbuminuria and time of postoperative reading:-

There was statistically significant difference between groups according to ACR t 0 to t24 specially at t6 which was highly significant in group II $(201.60 \pm 64.16)$ in relation to $(101.50 \pm 11.69)$ in group I with t-test ( 9.708 ) and p-value $<0.001$. 
Table (3): Comparison between groups according to ACR.

\begin{tabular}{lllll}
\hline & Group I & Group II & t-test & p-value \\
\hline ACR p & $17.75 \pm 4.79$ & $19.22 \pm 5.25$ & 1.308 & 0.195 \\
\hline ACR t0 & $23.50 \pm 4.99$ & $26.22 \pm 3.77$ & 2.751 & $\mathbf{0 . 0 0 7}$ \\
\hline ACR t6 & $101.50 \pm 11.69$ & $201.60 \pm 64.16$ & 9.708 & $<\mathbf{0 . 0 0 1}$ \\
\hline ACR t24 & $53.5 \pm 11.69$ & $81.22 \pm 49.00$ & 3.480 & $<\mathbf{0 . 0 0 1}$ \\
\hline
\end{tabular}

t: Independent Sample t-test

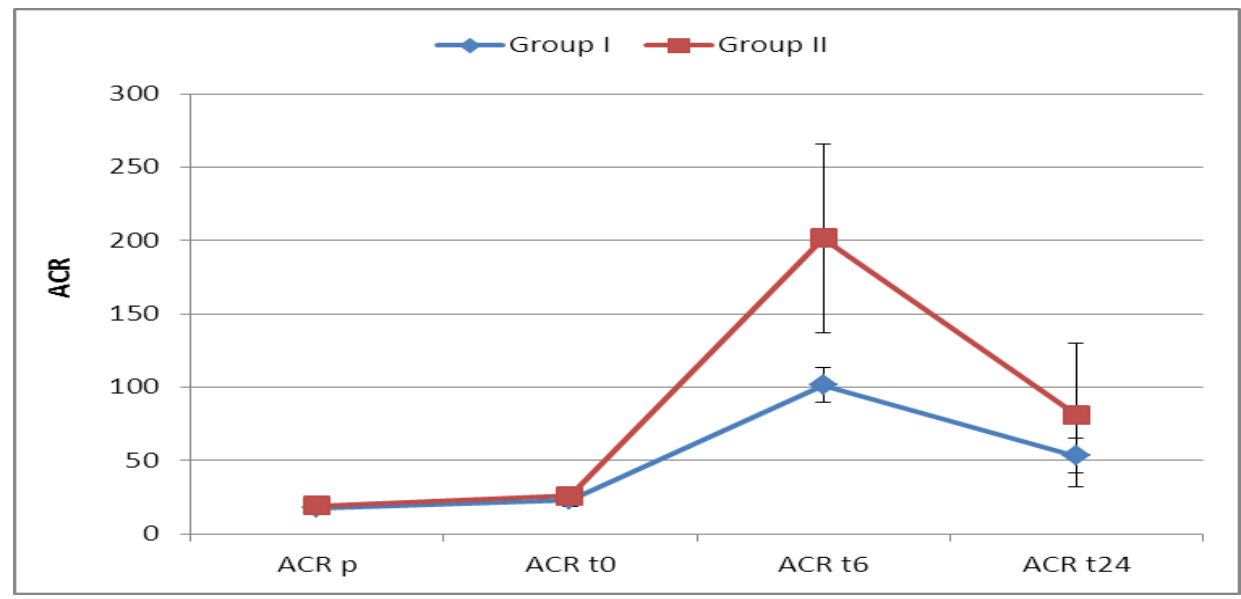

Figure (1): Comparison between groups according to ACR

\section{V) APACHE score and severity of illness:-}

There was highly statistically significant difference between groups according to APACHE score (15.00 \pm 2.72 in group II in relation to $9.00 \pm 2.19$ in group I for APACHE 24, $60.60 \pm 17.58$ in group II in relation to $45.52 \pm 12.00$ in group I for APACHE 48, $94 \pm 5.88$ in group II in relation to $66.70 \pm 4.59$ in group I for APACHE 72, p-value <0.001.

Table (4): Comparison between groups according to APACHE score.

\begin{tabular}{lllll}
\hline & Group I & Group II & t-test & p-value \\
\hline APACHE 24 & $9.00 \pm 2.19$ & $15.00 \pm 2.72$ & 10.876 & $<\mathbf{0 . 0 0 1}$ \\
\hline APACHE 48 & $45.52 \pm 12.00$ & $60.60 \pm 17.58$ & 4.481 & $<\mathbf{0 . 0 0 1}$ \\
\hline APACHE 72 & $66.70 \pm 4.59$ & $94 \pm 5.88$ & 23.147 & $<\mathbf{0 . 0 0 1}$ \\
\hline
\end{tabular}

t: Independent Sample t-test 


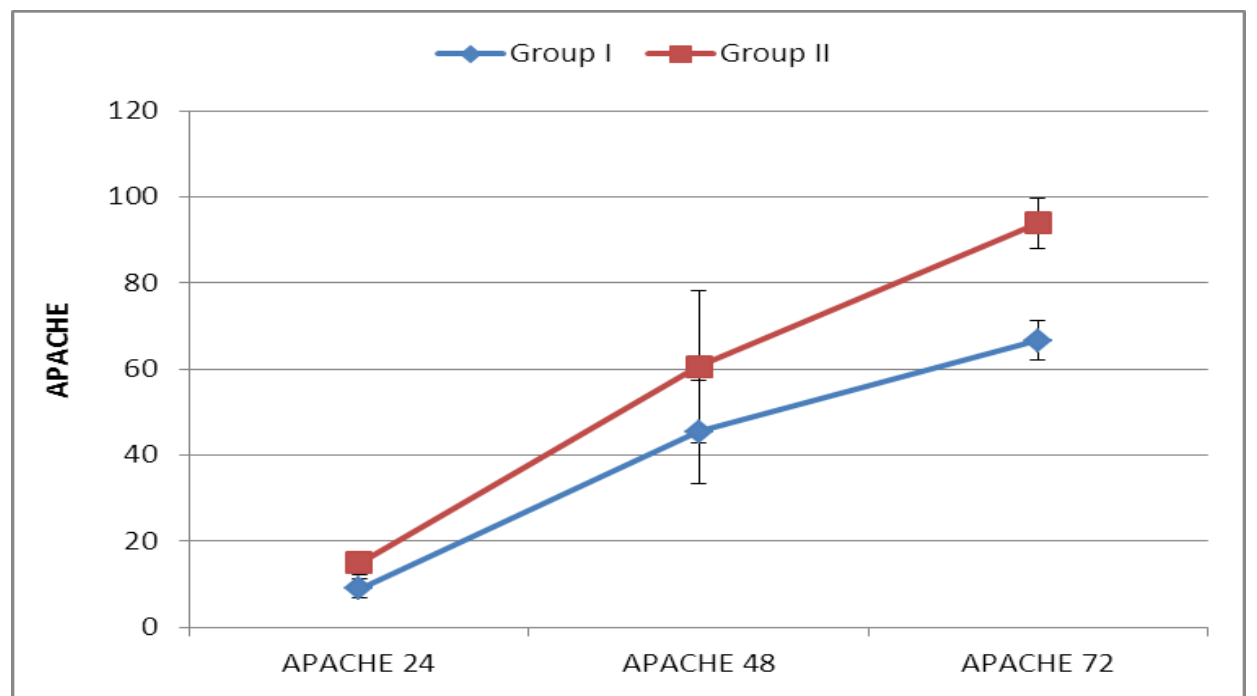

Figure (2): Comparison between groups according to APACHE score.

VI) SOFA score and severity of illness:-

There was highly statistically significant difference between groups according to SOFA score (6.15 \pm 3.86 in group II in relation to $2.4 \pm 1.70$ in group I for SOFA1, $12.75 \pm 2.61$ in group II in relation to $6.44 \pm 1.76$ in group I for SOFA2, $17.40 \pm 1.70$ in group II in relation to10.00 \pm 1.43 in group I for SOFA3, p-value $<0.001$.

Table (5): Comparison between groups according to SOFA score.

\begin{tabular}{lllll} 
& Group I & Group II & t-test & p-value \\
\hline SOFA 1 & $2.4 \pm 1.70$ & $6.15 \pm 3.86$ & 5.261 & $<\mathbf{0 . 0 0 1}$ \\
\hline SOFA 2 & $6.44 \pm 1.76$ & $12.75 \pm 2.61$ & 12.677 & $<\mathbf{0 . 0 0 1}$ \\
\hline SOFA 3 & $10.00 \pm 1.43$ & $17.40 \pm 1.70$ & 21.068 & $<\mathbf{0 . 0 0 1}$ \\
\hline
\end{tabular}

t: Independent Sample t-test

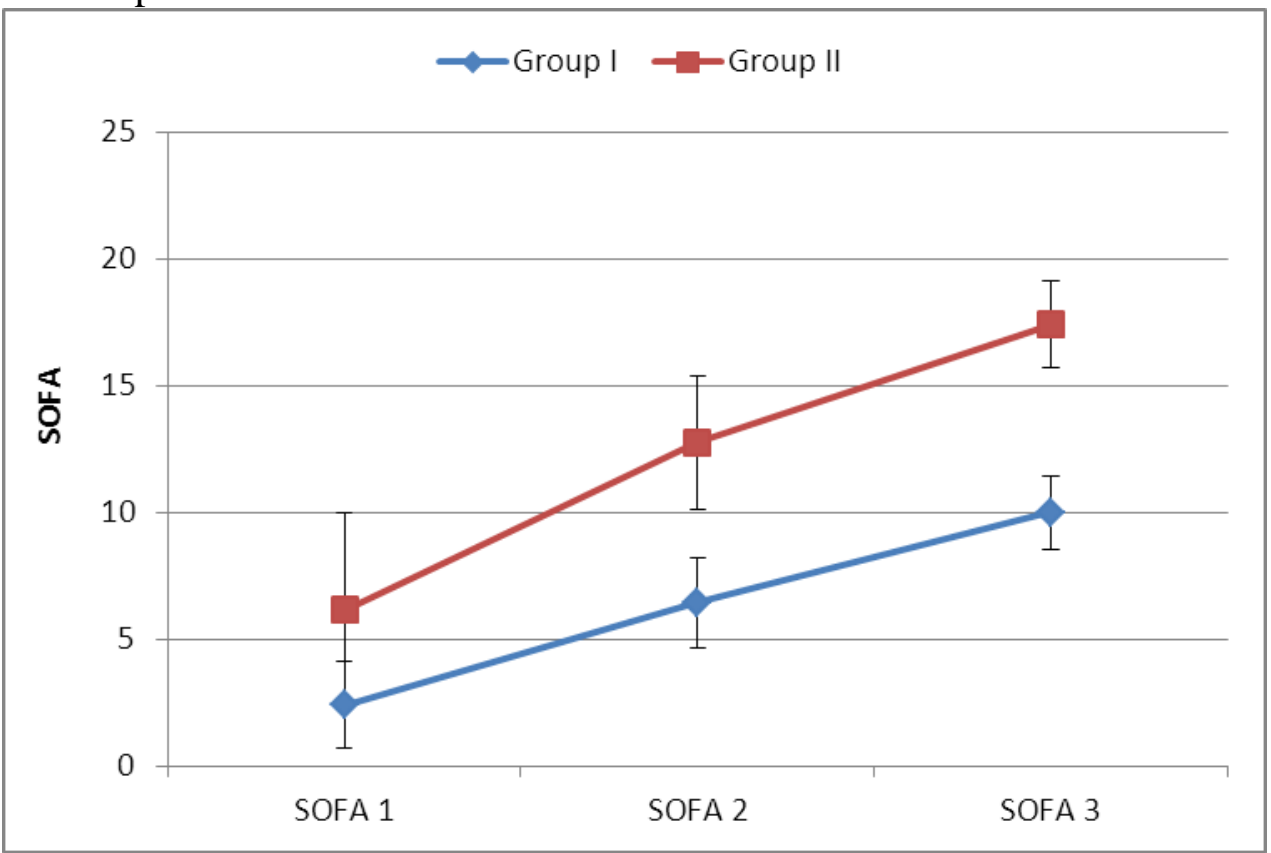

Figure (3): Comparison between groups according to SOFA score.

VII) Difference between two groups according to outcome:- 
There was statistically significant difference between groups according to outcome (discharge was 25 $(62.5 \%)$ in group II in relation to $35(87.5 \%)$ in group I while death was $15(37.5 \%)$ in group II in relation to $5(12.5 \%)$ in group I) p-value 0.020 .

Table (6): Comparison between groups according to outcome.

\begin{tabular}{lllll}
\hline OUTCOME & Group I & Group II & x2 & p-value \\
\hline Discharge & $35(87.5 \%)$ & $25(62.5 \%)$ & \multirow{2}{*}{5.400} & \multirow{2}{*}{$\mathbf{0 . 0 2 0}$} \\
\hline Death & $5(12.5 \%)$ & $15(37.5 \%)$ & &
\end{tabular}

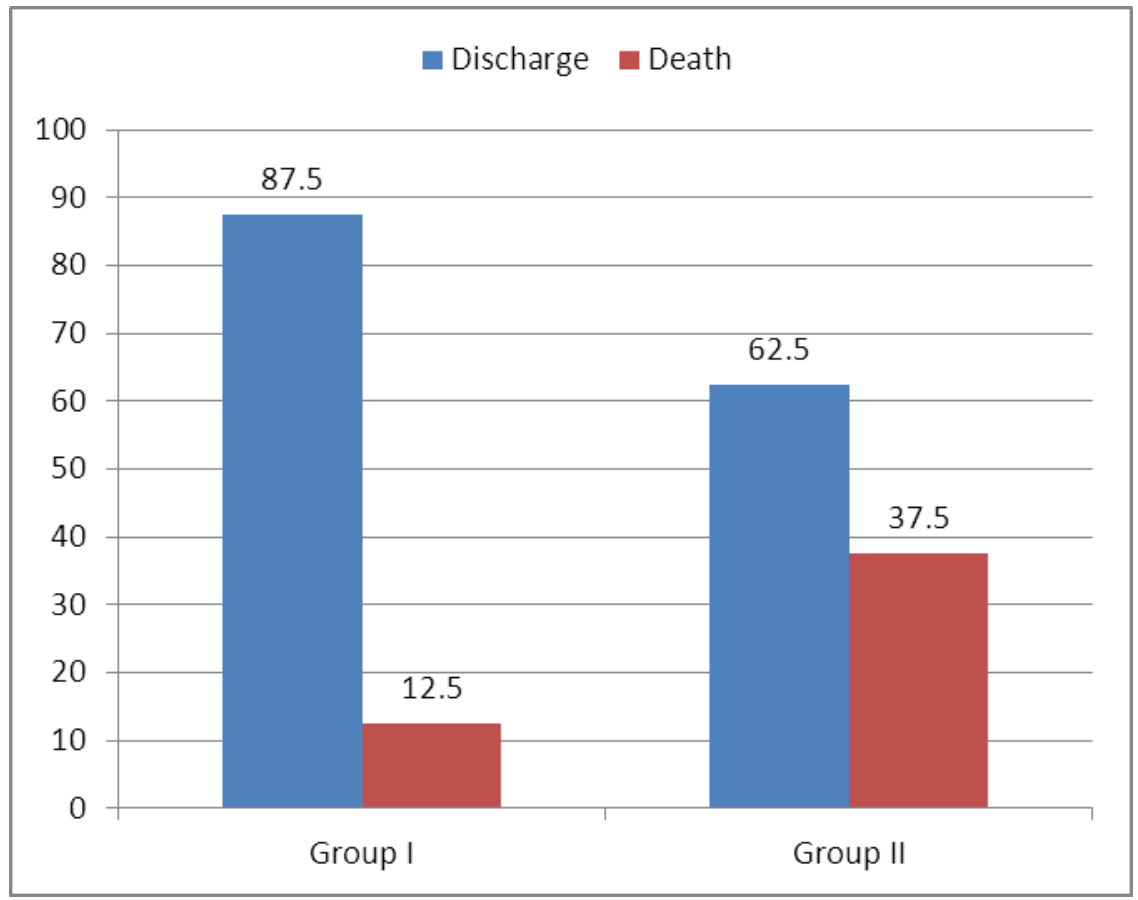

Figure (4): Comparison between groups according to outcome.

\section{DISCUSSION}

Sepsis is common in the critically ill patients ${ }^{[13]}$. Rapid induction of microalbuminuria levels occur within a few hours of an inflammatory assault as compared to relatively delayed inductions of procalcitonin (PCT) and C-reactive protein (CRP) ${ }^{[14]}$.

We tried in this study to investigate the value of Microalbuminuria as a prognostic tool for illness severity, clinical course and mortality in the critically ill surgical patients. Several studies in various groups of critically ill patients have used microalbuminuria as a significant prognostic marker of illness severity and mortality in the ICU ${ }^{[14]}$.

Increased frequency of microalbuminuria in critically ill patients is caused by global profound endothelial dysfunction arising from the effects of inflammatory mediators, released during the severe inflammatory assaults that are conjoined with critical illnesses leading to systemic increase in capillary permeability ${ }^{[15]}$.

Due to altered glomerular endothelial permeability in both kidneys, high amounts of microalbumin will escape into the glomerular filtrate. Reabsorptive capacity of the renal 
tubules for albumin from the ultrafiltrate exceeded beyond its capacity, leading to increased excretion of microalbumin in the urine $^{[8]}$.

Microalbuminuria would be an excellent marker for the early identification of patients with high risk of morbidity and mortality. This would allow the ICU physician to decide early therapeutic interventions in patients most likely to get benefit from its use, especially in situations of financial limitations ${ }^{[8]}$.

measurment of the amount of microalbumin excreted in urine, expressed as (ACR) ${ }^{[3]}$, is a simple, reliable, and cheap test that adjusts for different urine concentrations among ICU patients and avoids the need for a timed urine collection, Moreover it can be done while the patient in bed and results can be gained as early as $15 \mathrm{~min}^{[2]}$.

On the other hand, the ICU prognostic scores such as SOFA and APACHE scores requires variable data collection within 24 hours and involve meticulous statistical analyses to anticipate mortality as an outcome.

In our study, microalbuminuria on admission, was found to be a weak predictor of morbidity and mortality $(P=0.007)$, and therefore neither microalbuminuria on admission can be a useful tool to predict morbidity and mortality in critically ill surgical patients nor APACHE and SOFA scores.

After 6 hours, microalbuminuria found to be a good predictor of SIRS $(P<0.001)$, which indicates that, microalbuminuria during the first 6 hours is a good predictor of outcome.

Our findings are consistent with that of MacKinnon and colleagues ${ }^{[3]}$, they recruited 50 patients and then calculated probability of death for ACR and suggested that a rapid prediction of outcome can be gained within a window of 6 $\mathrm{h}$ of ICU admission.

Gosling and colleagues ${ }^{[2]}$, reported that elevated ACR level can predict death and APACHE score did.

We also demonstrated that within the first 6 hours, ACR was significantly high in the nonsurvivors compared with survivors $(\mathrm{P}<$
0.001).This finding correlate with previous studies done by Gosling et al., 2003; ${ }^{[2]}$ Thorevska et al., 2003, ${ }^{[16]}$ indicating that failure of ACR to decrease is combined with increased ICU mortality.

The choice of 6 hours as the timing for sampling was selected to assess the earliest time of rising of ACR in the non survivors as comparing with the survivors.

A declining trend of ACR after 6 hours from ICU admission may indicate a change of endothelial dysfunction towards the better and improvement in Organ function and a positive response to the initial management ${ }^{[8]}$.

In septic postsurgical patients, ACR found to correlate well with the sequential organ failure assessment scores as found by De Gaudio AR and colleagues ${ }^{[17]}$. But in our study ACR found to precede the change in SOFA score. SOFA score was found to start increasing non significantly at the first day and daily for 3 days in the first group and significantly from the first day and in the following 2 days in the second group.

We could not find an association between admission ACR and outcome. This contradicts Gosling's findings in 2003 where he found that elevated ACR within $15 \mathrm{~min}$ of the patients admission to ICU correlated with mortality ${ }^{[2]}$.Most authors support the principle that ACR measured on ICU admission, in particular within 6 hours from the postoperative time, to be most reflective of illness severity and prediction of outcome.

On the other hand, our study demonstrated that ACR in critically ill surgical patients does not correlate with ICU stay. This finding advocates with previous studies of Gosling et $\mathrm{al}^{[2]}$.

Lastly, when comparing microalbuminuria with other prognostic factors like Procalcitonin PCT, we can notice that Procalcitonin differs from other biomarkers of sepsis. Serum PCT serum levels are detectable as early as 3-4 hours after invasion of bacterial infection ${ }^{[18]}$, which is much earlier than the increase in the C-reactive protein level or microalbuminuria but, we can 
notice that the time of rise of microalbuminuria in our study was 6 hours which is not too much different from procalcitonin. Also microalbuminuria cost as a test is too much cheaper than procalcitonin.

The effect of an event rather than the current medical condition such as chronic obstructive airways disease, bronchial asthma or others that lead to ICU admission will be variable. These patients may stay a long time in ICU, not necessarily due to the acute insult, but because of the comorbidities. Recently, it has been declared that obesity was associated with increasing risk of renal injury ${ }^{[19]}$.

\section{CONCLUSION}

Microalbuminuria an easy, rapid and simple tool has a prognostic value in surgical sepsis, with particular regard to its role in predicting illness severity, clinical course, primary outcome (sepsis) and secondary outcome (mortality).

\section{Limitation to the study:}

Financial elements was an important factor due to the high cost of the tests used in the study, also time factor was of limitating importance because post-operative surgical cases was not common as non-surgical cases.

\section{RECOMMENDATION}

Future studies including wider clinical categories and independent perplexers of microalbuminuria are envisaged to evaluate the strength of the ACR test. Also, more studies are needed to compare between microalbuminuria and other prognostic values in intensive care environment. It will also be important to study the kinetics of microalbuminuria in different groups of sepsis and SIRS and to evaluate its usefulness on a "sepsis prediction panel" in combination with other sepsis biomarkers.

\section{REFERENCES}

1.B.A. Dubaybo, Microalbuminuria: simple, inexpensive, and dynamic marker of critical illness, Chest 120 (2001) (6), pp. 1769-1771.

2.Gosling P. Microalbuminuria: a marker of systemic disease. Br J Hosp Med 1995; 54(6): 285-290.

3.MacKinnon KL, Molnar Z, Lowe D, Watson ID, Shearer E. Use of microalbuminuria as a predictor of outcome in critically ill patients. $\mathrm{Br}$ J Anaesth 2000;84:239-41.

4.D. Dutta, S. Choudhuri, S. A. Mondal, S. Mukherjee, and S. Chowdhury, "Urinary albumin: creatinine ratio predicts prediabetes progression to diabetes and reversal to normoglycemia: role of associated insulin resistance, inflammatory cytokines and low vitamin D," Journal of Diabetes, vol. 6, no. 4, pp. 316-322, 2014.

5.Abid, Q. Sun, K. Sugimoto et al., Predictive value of microalbuminuria in medical ICU patients: results of a pilot study, Chest 120 (2001) (6), pp. 1984-1988.

6.Levy MM; Fink MP; Marshall JC et al., SCCM/ESICM/ ACCP/ATS/SIS. 2001 SCCM/ESICM/ACCP/ATS/SIS International Sepsis Definitions Conference. Crit Care Med. 2003;31:1250-6.

7.Gosling P. Microalbuminuria: a marker of systemic disease. BrJ Hosp Med 1995; 54(6): 285-290.

8.Surupa B, Chaudhuri S, Bhattacharyya $M$ et al., Microalbuminuria: an inexpensive, non invasive bedside tool to predict outcome in critically ill patients, Indian Journal of Clinical Biochemistry, 2010 / 25 (2) 146-152.

9. L. Selistre, V. De Souza, P. Cochat et al., "GFR estimation in adolescents and young adults," Journal of the American Society of Nephrology, vol. 23, no. 6, pp. 989-996, 2012.

10.Zimmerman J, Kramer A, McNair A et al., Acute Physiology and Chronic Health Evaluation(APACHE). IV: ICU length of stay benchmarks for today's critically ill patients. Crit Care Med 2006; 34:2517-2529.

11.Ferreira F, Bota D, Bross et al., Serial evaluation of the SOFA score to predict outcome in critically ill patients. JAMA 2001; 286: 17548.

12.Yew WS and Pal SK (2006): Correlation of microalbuminuria and outcome in patients with extensive burns. British Journal of Anaesthesia 97 (4): 499-502.

13.Becker KL, Snider R, Nylen ES. Procalcitonin assay in systemic inflammation, infection, and sepsis: Clinical utility and limitations. Crit Care Med 2008;36:941-52.

14. Gopal S, Carr B, Nelson P. Does microalbuminuria predict illness severity in critically ill patients on the intensive care unit? A 
systematic review. Crit Care Med 2006;34:180510.

15. Dziedzic T, Slowik A, Szczudlik A. Urine albumin excretion in acute ischaemic stroke is related to serum interleukin-6. Clin Chem Lab Med 2004; 42(2):182-5

16. Thorevska, R. Sabahi, A. Upadya et al., Microalbuminuria in critically ill medical patients: prevalence, predictors, and prognostic significance, Crit Care Med 31 (2003) (4), pp. 1075-1081

17. De Gaudio AR, Adembri C, Grechi S, Novelli

GP. Microal- buminuria as an early index of impairment of glomerular perme- ability in postoperative septic patients. Int Care Med 2000; 26: $1364-8$

18. Becker KL,

Snider R, Nylen ES. Procalcitonin assay in systemic inflammation, infection, and sepsis: clinical utility and limitations. Crit Care Med 2008;36:941-52.

19. N. Xiao, T. M. Jenkins, E. Nehus et al., "Kidney function in severely obese adolescents undergoing bariatric surgery," Obesity, vol. 22, no. 11, pp. 2319-2325, 2014. 